\title{
GPT intern
}

\section{Neues aus dem Beirat der korporativen Mitglieder}

Der Beirat der korporativen Mitglieder der GPT tagte am 16. Mai 2017 unter der Leitung seines wiedergewählten Vorsitzenden Dr. Christian Nauert in Köln. Einen Schwerpunkt der Diskussion bildeten aktuelle Fragen zu Pyrrolizidinalkaloiden (PA), insbesondere zur Umsetzung der Bekanntmachung des BfArM vom 1. März 2016 und die sich hieraus ergebenden Fragen, die im November 2016 mit dem BfArM diskutiert und in einem Empfehlungspapier der Verbände BAH und BPI erläutert worden waren. Das Europäische Arzneibuch hat neuerdings eine Arbeitsgruppe eingerichtet, die eine Rahmenmonografie für eine Analysenmethode zur PA-Bestimmung erarbeiten soll. Neuigkeiten gibt es auch bei den laufenden Forschungsprojekten zu den durch Unkräuter ins Erntegut gelangenden PA, z.B. bei der Erfassung der Unkrautflora in Arzneipflanzenbeständen und der Erarbeitung sogenannter „Unkrautsteckbriefe“. An der Universität Kaiserslautern wird ein Projekt durchgeführt, das die Hepato- und die Genotoxizität von neuen ausgewählten Alkaloiden in vitro untersucht, auf eine Berechnung sachgerechterer Grenzwerte für PA abzielt und von Mitgliedsfirmen der Kooperation Phytopharmaka gefördert wird.

Der Beirat der korporativen Mitglieder diskutierte auch die Aktivitäten der EUKommission in Bezug auf den „Fitness Check" der europäischen Health ClaimsVerordnung für Nahrungsergänzungsmittel (ausführlicher Beitrag dazu folgt in Heft 4). Die Online-Befragungen von Verbänden ist abgeschlossen, derzeit laufen sowohl eine Konsultation der Öffentlichkeit als auch kleiner und mittlerer Unternehmen. Der Beirat hielt fest, dass die

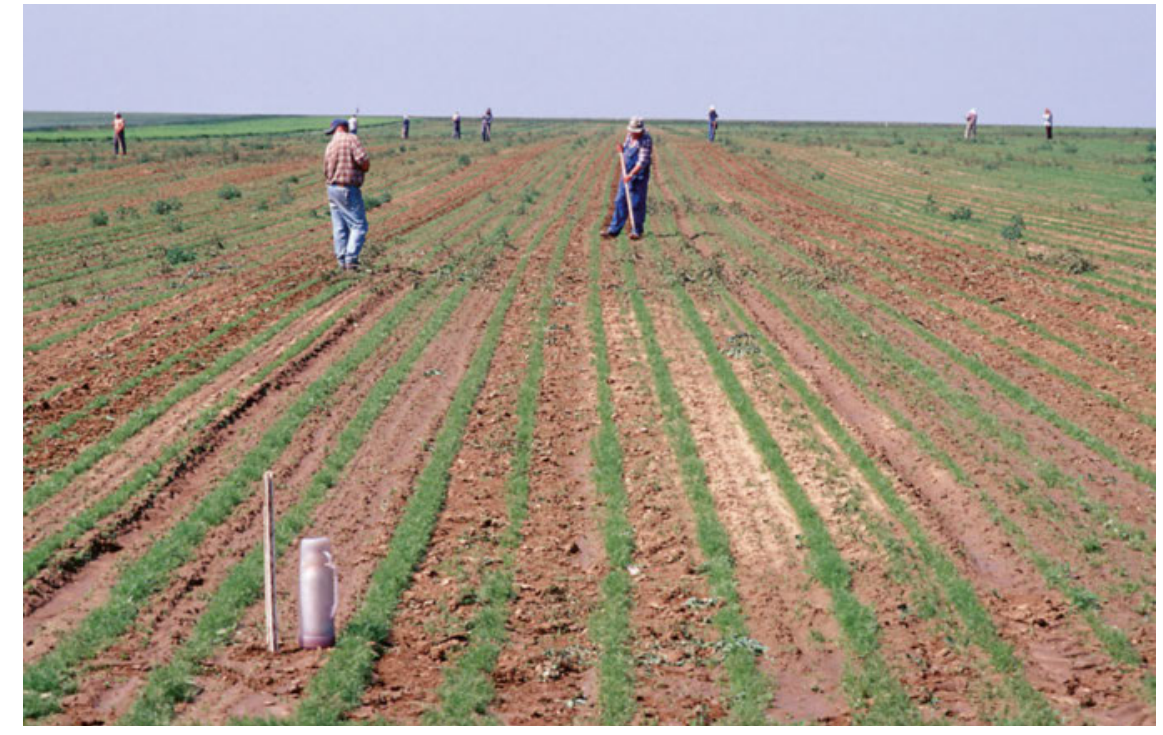

Viel Handarbeit: Beikrautregulierung gegen PA-Eintrag. (c) BLE, Bonn/Foto: Thomas Stephan. www.oekolandbau.de

GPT sich als Sachwalter des pflanzlichen Arzneimittels in der Vergangenheit eindeutig zur Beibehaltung der gesetzlichen Kriterien zur Bewertung der Produktaussagen pflanzlicher Nahrungsergänzungsmittel positioniert hat.

\section{HMPC: Neue Monografien in der Pipeline}

Vorgestellt wurden weiterhin einige Dokumente des Herbal Medicinal Products Committee (HMPC) wie z.B. die in Arbeit befindlichen Monografien zu Mariendistelfrüchten, Bärentraubenblättern, Steinkleekraut, Pfefferminzblättern und Schwarzen Johannisbeerblättern sowie der Entwurf der Bewertung von Sojabohnen, der hinsichtlich einer MonografieErstellung zu einer negativen Schlussfolgerung kommt. Neu sind die Aufrufe zur
Einreichung von Daten für 3 Tee-Kombinationen, für die - ähnlich den deutschen Standardzulassungen - Monografien mit einem „Bausteinprinzip“ für die Kombination einzelner Teedrogen erstellt werden sollen.

\section{Stellenwert nicht-interventio- neller Studien}

Die nunmehr verabschiedete wissenschaftliche Anleitung der EMA zum Design von Post-Authorization Efficacy Studies (PAES) greift u.a. die Meinung der GPT auf, dass nicht-interventionelle Studien durchaus einen Platz bei den Wirksamkeitsstudien haben können. In Bezug auf die Evidenz bei der Anwendung pflanzlicher Arzneimittel bei Kindern hat der Beirat für die GPT ein Positionspapier „Evidence Generation in the Paediatric 
Population - Extrapolation“ erstellt, das auf der GPT-Homepage sowie in einer Fachzeitschrift publiziert und ebenfalls bei Veranstaltungen als Poster präsentiert werden soll.

Das neue Concept Paper der EMA „Guideline on the use of pharmacokinetics in the development of medicinal products for children“ enthält den Ansatz, dass nicht nur Pharmakokinetik-Daten, sondern auch Daten aus Beobachtungsstudien bei der Bewertung von Kinderarzneimitteln Verwendung finden können und befürwortet grundsätzlich das Extrapolationskonzept. Auch hierzu wird die GPT eine Kommentierung vornehmen und dabei auf die Besonderheiten pflanzlicher Arzneimittel hinweisen.
Die nächste Sitzung des Beirats der korporativen Mitglieder findet im November 2017 statt.

Dr. Barbara Steinhoff

steinhoff@phytotherapie.de 